\title{
СПЕКТР ВХОДНОГО ТОКА ГРУППЫ УПРАВЛЯЕМЫХ ВЫПРЯМИТЕЛЕЙ, РАССТРОЕННЫХ ПО ТОКУ ПРИ ЗАДАННОМ РАСХОДЕ ГАЗА ПИТАЕМЫХ ПЛАЗМОТРОНОВ
}

T. TOMSON. JAAVA GAASIKULU JA VOOLU HALVETEGA PLASMATRONE TOITVATE JUHITAVATE ALALDITE GRUPI SISENDVOOLU SPEKTER

T. TOMSON. THE INPUT CURRENT SPECTRUM OF A GROUP OF CONTROLLED RECTIFIERS FEEDING PLASMATRONS AT FIXED GAS FLOW AND DEFLECTED VALUE OF ANY SINGLE CURRENT

\section{(Представил Н. Эпик)}

В [ $\left.{ }^{1}\right]$ рассматривается групповая работа 6-пульсных управляемых выпрямителей (УВ), питающих плазмотроны. Показано, что в случае расстройки по углу управления $\alpha$ при постоянном токе $I$ можно достичь более благоприятного гармонического состава входного тока. Однако это достигается за счет нарушения симметрии газового режима, например, в многоструйном химическом реакторе, что не всегда оправдано. Поэтому исследуем режим групповой работы, который появляется при расстройке токов на $\Delta I$ в окрестности рабочей точки $U, I$ при номинальном расходе газа $G_{\text {ном }}=$ const $\left[{ }^{2}\right]$. Из-за конечного дифференциального сопротивления ВАХ-плазмотрона при расстройке тока па $\Delta I$ появляется расстройка рабочего напряжения на

$$
\Delta U=-\Delta I U / 3 I \text {, }
$$

откуда для 6-пульсного УВ с регулировочной характеристикой $U=$ $=U_{d 0} \cos \alpha$ найдем расстройку по углу управления

$$
\begin{gathered}
\Delta_{\alpha}=\chi \Delta I / I, \\
\chi=U / U_{d 0} \sqrt{1-\left(U / U_{d 0}\right)^{2}} .
\end{gathered}
$$

где

Временная диаграмма тока при общепринятых упрощающих допущениях изображена на рисунке, $a$. K этим допущениям относятся мгновенпая коммутация вентилей $\gamma=0$ и полностью сглаженный выходной ток $I(t)=I=$ const. На упомянутом рисунке сплошной линией показан импульс входного тока нерасстроенного по току УВ, пунктирной линией - импульс тока УВ с повышенным на $\Delta I$ значением и штрих-пунктирной - импульс тока УВ с пониженным на $\Delta I$ значением тока. Ввиду преднамеренности расстройки $\Delta I$ является детерминированной величиной, задаваемой произвольно. Для определения спектра суммарного тока, используя принцип суперпозиции $\left[{ }^{1}\right]$, определим сигнал поправки, который отличает каждый расстроенный по амплитуде (и по фазе) частный сигнал от нерасстроенного. Этот сигнал поправки показан на рисунке в части б. Заметим, что для каждой пары сигналов, расстроенных по амплитуде, существуют взаимно-компенсирующие 
части, которые определяют нулевое мгновенное значение суммарного сигнала поправки. Это позволяет не учесть эти заштрихованные части сигналов и предъявить суммарный сигнал поправки в виде, который показан на рисунке в части 8 .
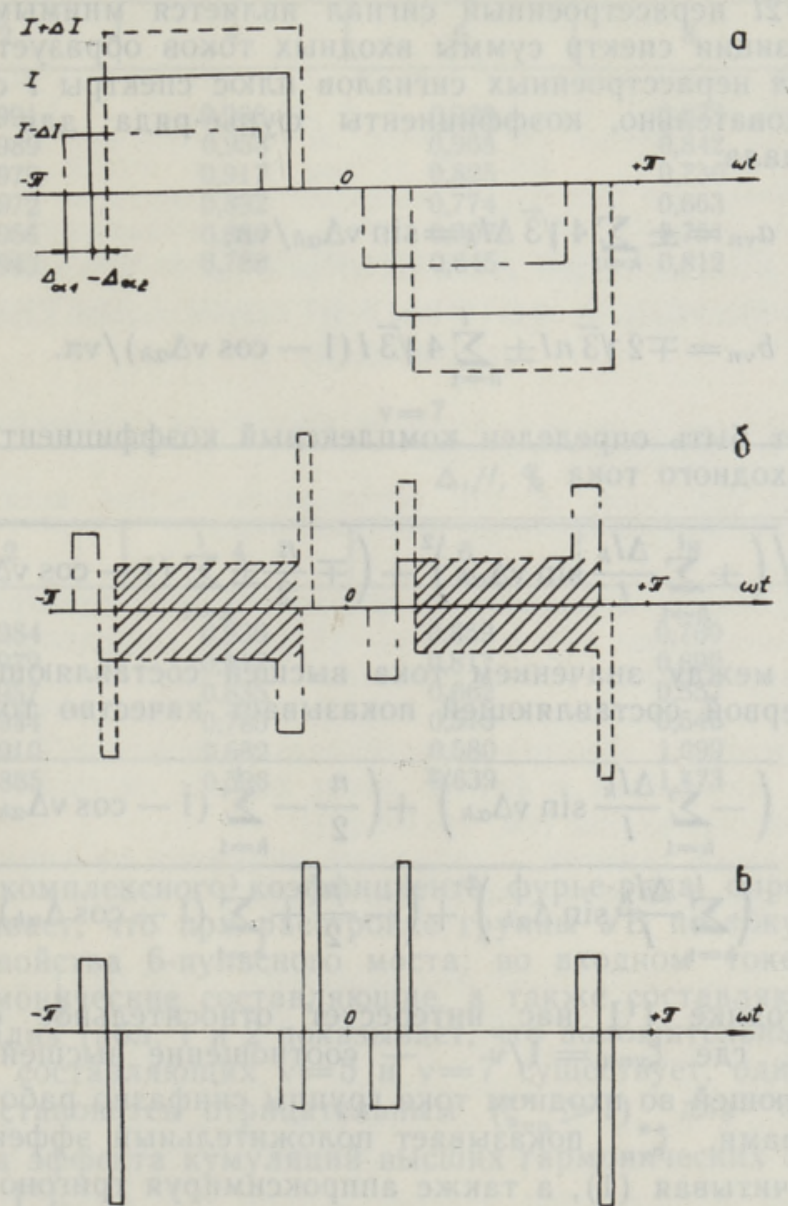

Временная диаграмма импульсов входного тока одной фазы при расстройке выпрямленных токов $I, I \pm \Delta I: a-$ импульсы тока; $\sigma$ - частные сигналы поправки и в - суммарный сигнал поправки.

Для группы $n$ синфазно управляемых УВ могут быть определены в результате фурье-анализа следующие компоненты в случае $v$-й гармонической составляющей

$$
\begin{aligned}
& a_{v}=0, \\
& b_{v}=c_{v}=4 n I \sin v \frac{\pi}{2} \sin v \frac{\pi}{3} / v \pi=\mp 2 \sqrt{3} I / v \pi .
\end{aligned}
$$

Для сигнала поправки каждой $l$ пары УВ в группе из $n$ УВ, расс-роенных по току, могут быть определены следующие коэффициенты фурьеряда:

$a_{v}=8 \Delta I \sin v \frac{\pi}{2} \sin v \frac{\pi}{3} \sin v \Delta_{\alpha} / v \pi= \pm 4 \sqrt{3} \Delta I \sin v \Delta_{\alpha} / v \pi$,

$b_{v}=8 I \sin v \frac{\pi}{2} \sin v \frac{\pi}{3}\left(1-\cos v \Delta_{\alpha}\right) / v \pi= \pm 4 \sqrt{3} I\left(1-\cos v \Delta_{\alpha}\right) / v \pi$. 
В формулах (2) и (3) верхние знаки действителььны для $v=1$, нижние для $v \in\{5,7\}$.

Для нечетного числа нагрузок $n=2 l+1$ один из токовых сигналов может быть рассмотрен как нерасстроенный сигнал, для четного числа нагрузок $n=2 l$ нерасстроенный сигнал является мнимым. По принципу суперпозиции спектр суммы входных токов образуется как сумма спектров $n$ нерасстроенных сигналов плюс спектры $l$ сигналов поправок. Следовательно, коэффициенты фурье-ряда для суммарного входного сигнала

$$
\begin{aligned}
& a_{v n}= \pm \sum_{k=1}^{l} 4 \sqrt{3} \Delta I_{k}=\sin v \Delta_{\alpha k} / v \pi, \\
& b_{v n}=\mp 2 \sqrt{3} n I \pm \sum_{k=1}^{l} 4 \sqrt{3} I\left(1-\cos v \Delta_{\alpha k}\right) / v \pi .
\end{aligned}
$$

По (4) может быть определен комплексный коэффициент фурье-ряда суммарного входного тока

$$
c_{v n}=\frac{4 \sqrt{3} I}{v \pi} \cdot \sqrt{\left( \pm \sum_{k=1}^{l} \frac{\Delta I_{k}}{I} \sin v \Delta_{\alpha k}\right)^{2}+\left(\mp \frac{n}{2} \pm \sum_{k=1}^{l}\left(1-\cos v \Delta_{\alpha k}\right)\right)^{2}} .
$$

Соотношение между значением тока высшей составляющей и значением тока первой составляющей показывает качество тока [ $\left.{ }^{1}\right]$.

$$
\zeta_{v n}=\frac{1}{v} \sqrt{\frac{\left(-\sum_{k=1}^{l} \frac{\Delta I_{k}}{I} \sin v \Delta_{\alpha k}\right)^{2}+\left(\frac{n}{2}-\sum_{k=1}^{l}\left(1-\cos v \Delta_{\alpha k}\right)\right)^{2}}{\left(\sum_{k=1}^{l} \frac{\Delta I_{k}}{I} \sin \Delta_{\alpha k}\right)^{2}+\left(-\frac{n}{2}+\sum_{k=1}^{l}\left(1-\cos \Delta_{\alpha k}\right)\right)^{2}}} .
$$

Согласно методике [ $\left.{ }^{1}\right]$ нас интересует относительнфе соотношение $\zeta_{v n}^{*}=\zeta_{v n} / \zeta_{v n n}$, где $\zeta_{v n \pi}=1 / v-$ соотношение высшей гармонической составляющей во входном токе группы синфазно работающих УВ. Другими словами, $\zeta_{v n}^{*}$ показывает положительный эффект расстройки. По (6), учитывая (1), а также аппроксимируя тригонометрические функции первыми членами их степенных рядов и представляя расстройки по току как кратные выбранного кванта по току $\Delta I_{k}=\sum_{i=1}^{k} \Delta_{i}$, можем вывести расчетную формулу

$$
\zeta_{v n}^{*} \approx \sqrt{\frac{\left(-v\left(\lambda \frac{\Delta_{i}}{I}\right)^{2}\right)^{2}+\left(n / \chi-v^{2} \chi\left(\lambda \frac{\Delta_{i}}{I}\right)^{2}\right)^{2} / 4}{\left(\left(\lambda \frac{\Delta_{i}}{I}\right)^{2}\right)^{2}+\left(-n / \chi+\chi\left(\lambda \frac{\Delta_{i}}{I}\right)^{2}\right)^{2}}} .
$$

В формуле (7) $\lambda$ - вспомогательная величина, которая определяется из соотношения

$$
\lambda=\sum_{k=1}^{l} \Delta I_{k} / \Delta_{i}
$$

Например, для $n=2, \lambda=3, n=3, \lambda=6$ и т. п. Рассчитанные по (7) значения $\zeta_{v n}^{*}$ для $v=5$ и $v=7$ показаны в табл. 1 и 2. Верхние цифры получены для $\lambda=0,483$ (при $U / U_{d 0}=0,5$ ), нижние для $\lambda=0,494$ (при $\left.U / U_{d 0}=0,65\right)$. 
$v=5$

\begin{tabular}{c|c|c|c|c|c}
\hline \multirow{2}{*}{$n$} & \multicolumn{5}{|c}{$\Delta_{i} / I, \%$} \\
\cline { 2 - 6 } & 2 & 4 & 6 & 8 & 10 \\
\hline \multirow{2}{*}{2} & 0,991 & 0,968 & 0,929 & 0,878 & 0,819 \\
& & 0,958 & 0,908 & 0,842 & 0,766 \\
3 & 0,989 & 0,917 & 0,825 & 0,730 & 0,691 \\
& 0,979 & 0,892 & 0,774 & 0,663 & 0,665 \\
4 & 0,972 & 0,836 & 0,707 & 0,751 & 1,120 \\
& 0,943 & 0,788 & 0,645 & 0,812 & 1,382
\end{tabular}

Таблища 2

$v=7$

\begin{tabular}{c|c|c|c|c|c}
\hline \multirow{2}{*}{$n$} & \multicolumn{5}{|c}{$\Delta_{i} / I, \%$} \\
\cline { 2 - 6 } & 2 & 4 & 6 & 8 & 10 \\
\hline \multirow{2}{*}{2} & 0,984 & 0,936 & 0,859 & 0,760 & 0,652 \\
& 0,979 & 0,917 & 0,817 & 0,690 & 0,563 \\
3 & 0,957 & 0,835 & 0,662 & 0,557 & 0,747 \\
& 0,944 & 0,785 & 0,573 & 0,546 & 0,958 \\
4 & 0,910 & 0,682 & 0,580 & 1,099 & 2,04 \\
& 0,885 & 0,596 & 0,639 & 1,473 & 2,710
\end{tabular}

Анализ комплексного коэффициента фурье-ряда, определяемого по (3), показывает, что при расстройке группы УВ по току сохраняются основные свойства 6-пульсного моста: во входном токе отсутствуют четные гармонические составляющие, а также составляющие, кратные к трем. Анализ табл. 1 и 2 показывает, что положительный эффект для подавления составляющих $v=5$ и $v=7$ существует, однако он незначителен и становится отрицательным $\left(\zeta_{v n}^{*}>1\right)$ для больших расстроек из-за эффекта кумуляций высших гармонических составляющих, аналогично [ $\left.{ }^{1}\right]$.

\section{Л И Т Е Р А Т У РА}

1. Томсон T. // Изв. АН ЭССР. Физ. Матем., 1984, 33, № 3, 339-349.

2. Тамкиви П. И., Томсон Т. И. // Изв. СО АН СССР. Сер. техн. наук, 1983, № 3, вып. 3, 77-82.

Ннститут термофизики и электрофизики Академии наук Эстонской ССР

Поступила в редакцию $17 / \mathrm{V} 1988$ 